\title{
Riutilizzo delle acque: cosa sono le clorammine e qual è il loro ruolo nei trattamenti avanzati?
}

\author{
Rosanna Feola ${ }^{1}$ \\ ${ }^{1}$ SEEDotti dall'Ambiente
}

\begin{abstract}
Il riutilizzo delle acque assume al giorno d'oggi una rilevanza sempre maggiore a causa della crescente domanda e della scarsità di acqua potabile. Sono stati effettuati diversi studi ed implementanti diversi sistemi al fine di ridurre i contaminanti dai reflui. Ai processi tradizionali si aggiungono processi innovativi ed avanzati che permettono di raggiungere risultati sempre più performanti. II processo che verrà illustrato in questo studio è uno dei trattamenti di ossidazione avanzata UV-basati, anche detti (UV/AOPs), in particolare I'UV/perossido di idrogeno. Sarà valutato l'impatto delle monoclorammine e delle diclorammine durante il processo UV/perossido di idrogeno per la rimozione del diossano. Le monoclorammine e le diclorammine sono dei composti del cloro che vengono aggiunti per prevenire il fouling delle membrane. Le membrane sono utilizzate come pre-trattamento all'UV/perossido di idrogeno in quanto permettono di effettuare microfiltrazione e osmosi inversa, ovvero di separare fisicamente il refluo dalle sostanza di scarto, ed è per questo motivo che sono spesso soggette a fouling ovvero si sporcano e si intasano. Il diossano D-1,4 è un contaminante che si trova nelle acque reflue poiché presente in alcuni prodotti per la cura personale ed utilizzato in alcuni processi industriali. In questo studio si vedrà come l'introduzione delle cloroammine influisca sulla performance del processo di ossidazione avanzata per rimuovere il diossano.
\end{abstract}

\section{Trattamenti avanzati per il riutilizzo delle acque}

Il riutilizzo delle acque è diventato sempre più importante per alleviare la carenza idrica nelle regioni colpite da siccità. I cambiamenti climatici svolgono un ruolo importante in questa ottica causando squilibri e portando alla necessità di trovare soluzioni ${ }^{1}$.II trattamento di riutilizzo si compone di una sequenza di processi, Fig.1, ognuno con lo scopo di ridurre specifici contaminanti microbiologici, inorganici e organici presenti nelle acque reflue che avrebbero effetti negativi sulla salute dell'uomo e dell'ambiente ${ }^{2}$. In questo studio ci si concentra sul processo UV-basato di ossidazione avanzata 
(UV/AOP) utilizzato per la disinfezione e la rimozione dei contaminanti. Prima di esso bisogna prevedere dei pre-trattamenti con membrane che riducano la presenza di particelle che altrimenti interferirebbero successivamente. Durante questi pre-trattamenti (microfiltrazione e osmosi inversa) le membrane sono soggette a fouling, per prevenirlo si introducono le clorammine che sono dei composti che si formano quando si aggiunge il cloro nell'acqua da trattare. II cloro reagisce e forma monoclorammine oppure diclorammine in funzione di alcuni parametri del refluo, ad esempio il $\mathrm{pH}^{3}$. L'efficacia della cloraminazione sulla mitigazione dello sporcamento è stata dimostrata da diversi studi, in cui si vede come la presenza delle clorammine limiti la crescita dei batteri ${ }^{4}, 5$. Dal momento che entrambe le clorammine sono però piccole, si diffondono facilmente tramite le membrane e vengono trasportate allo step successivo UV/AOP. Questo step è necessario al fine di rimuovere il diossano che è un composto classificato come possibilmente cancerogeno. Esso trova larga applicazione sia nei prodotti per la cura personale sia in altri campi, ad esempio come solvente per le stampe $3 \mathrm{D}{ }^{6}$; è importante quindi prevedere la sua rimozione dalle acque di processo. Il diossano in presenza di perossido di idrogeno e UV si degrada. Si è visto che anche le cloroammine degradano il diossano. In letteratura sono presenti diversi studi per la rimozione del diossano ${ }^{7}$. Tramite il processo UV/clorammine si ottengono buone rimozioni di diossano se utilizzato il dosaggio ottimale di clorammina ${ }^{8}$. Anche in altri scenari, l'efficienza dell'UV/clorammina risulta paragonabile a quella dell'UV/perossido di idrogeno ${ }^{9}$. Altri studi hanno invece investigato la rimozione del diossano in presenza di UV/clorammine e perossisolfato mostrando ancora una volta come la presenza di clorammine sia benefica a specifici dosaggi 10 . Alcuni ricercatori hanno proposto metodi alternativi per la rimozione del diossano, ad esempio tramite I'utilizzo di alberi come il pioppo ${ }^{11}$, oppure con il riscaldamento a resistenza elettrica ${ }^{12}$. Non si è però mai considerata la rimozione tramite la simultanea presenza di perossido di idrogeno e clorammine con UV che sarà presentata nello specifico in questo articolo in riferimento allo studio ${ }^{13}$. Sarà descritta l'efficienza e il tipo di reazioni che si hanno in questo sistema più complesso ed in particolare l'effetto sulla rimozione del diossano. 


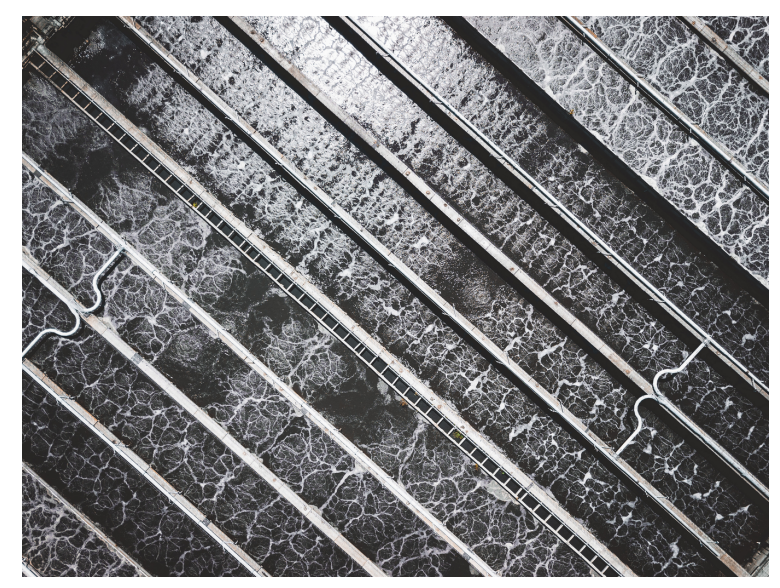

Figure 1: Impianto di trattamento reflui

\section{La rimozione del diossano con UV/H2O2 e clo- rammine}

Il processo UV/H2O2, dove $\mathrm{H} 2 \mathrm{O} 2$ è il perossido di idrogeno, rimuove i contaminanti grazie a dei radicali che si formano quando il perossido di idrogeno è sottoposto alla luce UV. II perossido di idrogeno trova applicazione per la rimozione di vari contaminanti, come ad esempio i coloranti tessili ${ }^{14}$. In particolare, invece, gli esperimenti che saranno descritti successivamente riguarderanno solo la rimozione del diossano: sono state preparate le miscele di acqua e diossano e si sono sperimentate diverse concentrazioni di clorammine per vedere come fossero influenzati i risultati durante il trattamento UV/H2O2. Bisogna considerare due aspetti fondamentali, ovvero che l'efficienza di rimozione diminuisce se:

- diminuiscono i radicali, poiché essi degradano il diossano;

- diminuisce la luce UV, poiché essa consente la formazione dei radicali.

I risultati hanno mostrato che al di sotto delle concentrazioni ottimali, la presenza delle cloroammine è stata benefica per la degradazione del diossano. Quando però il dosaggio ha superato il livello ottimale, le cloroammine hanno interferito con la produzione di radicali e quindi è diminuita l'efficienza di rimozione del diossano. In particolare, a bassi dosaggi di clorammine, le monoclorammine riescono a rimuovere il diossano il 60-80\% in più rispetto alle diclorammine; ad alti dosaggi di clorammine, le monoclorammine risultano meno efficienti rispetto alle diclorammine.

Si è poi visto che le miscele con una maggiore presenza percentuale di monoclorammine, rispetto alle diclorammine, migliora l'efficienza di degradazione del diossano quasi del 40\%. Ciò perchè al crescere delle diclorammine rispetto alle monoclorammine, diminuisce la produzione di radicali. 
Si è infine confrontato la rimozione del diossano sia nel sistema $\mathrm{UV} /$ perossido di idrogeno con sole monoclorammine sia nel sistema $\mathrm{UV} /$ perossido di idrogeno con sole diclorammine. I risultati dimostrano che nel composto con le sole monoclorammine anche l'aggiunta di una bassa concentrazione delle stesse porti ad una decrescita del tasso di rimozione del diossano, ciò perchè:

- diminuiscono i radicali, perchè reagiscono con le monoclorammine e non con il diossano;

- diminuisce la percentuale di luce UV, a causa della presenza delle monoclorammine.

Nel composto con le sole diclorammine invece, il tasso di rimozione del diossano si riduce di una quantità trascurabile rispetto alle monoclorammine, ciò perchè:

- le diclorammine eliminano molti meno radicali attivi rispetto alle monoclorammine;

- le diclorammine assorbono meno luce UV rispetto alle monoclorammine.

Pertanto, le diclorammine hanno avuto un impatto complessivo minore sulle prestazioni di UV/perossido rispetto alle monoclorammine. In Fig.2 si rappresenta sinteticamente il risultato dello studio.

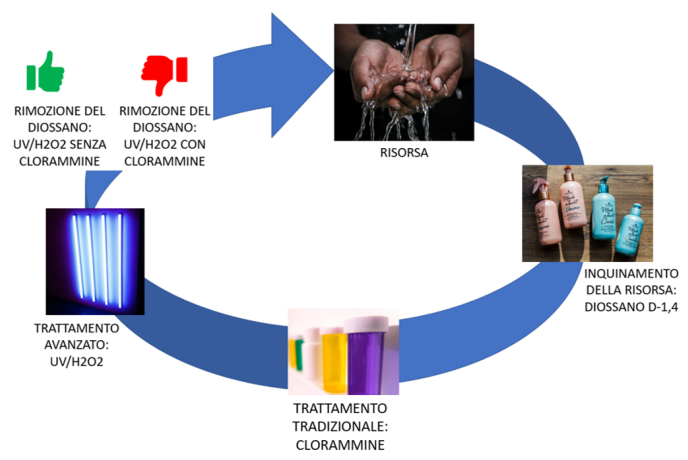

Figure 2: II ciclo del riutilizzo delle acque

\section{Conclusioni}

In conclusione, l'aggiunta di cloroammine nel trattamento UV/perossido può compromettere l'efficienza di rimozione del diossano, principalmente a causa dell'eliminazione dei radicali e degli effetti delle cloroammine come light screening. La rimozione delle cloroammine precedentemente al trattamento di UV/perossido può far crescere l'efficienza di trattamento. Nel 
caso in cui però, non fosse possibile la preventiva rimozione delle cloroammine, si potrebbe pensare di modificare alcuni parametri del refluo in modo tale da sfavorire la formazione delle monoclorammine e favorire invece quella delle diclorammine. Tutto ciò potrebbe portare a dei vantaggi nel trattamento, a patto però che si controlli la potenziale formazione di altri sottoprodotti più nocivi per la salute umana.

\section{References}

1. Hamed, Y. et al.. Climate impact on surface and groundwater in North Africa: a global synthesis of findings and recommendations. EuroMediterranean Journal for Environmental Integration 3, (2018).

2.Zuk, A. M., Tsuji, L. J. S., Nieboer, E., Martin, I. D. \& Liberda, E. N. Examining environmental contaminant mixtures among adults with type 2 diabetes in the Cree First Nation communities of Eeyou Istchee Canada. Scientific Reports 9, (2019).

3.Buxton, G. V., Bydder, M. \& Salmon, G. A. Reactivity of chlorine atoms in aqueous solution Part 1 The equilibrium CIMNsbd $+\mathrm{Cl}-\mathrm{Cl} 2$-. Journal of the Chemical Society Faraday Transactions 94, 653-657 (1998).

4.Yu, W., Xu, L., Graham, N. \& Qu, J. Pre-treatment for ultrafiltration: effect of pre-chlorination on membrane fouling. Scientific Reports 4, (2014).

5.Fujioka, T. et al.. Biofouling Mitigation by Chloramination during Forward Osmosis Filtration of Wastewater. International Journal of Environmental Research and Public Health 15, 2124 (2018).

6.He, Y., Wildman, R. D., Tuck, C. J., Christie, S. D. R. \& Edmondson, $\mathrm{S}$. An Investigation of the Behavior of Solvent based Polycaprolactone ink for Material Jetting. Scientific Reports 6, (2016).

7.Broughton, A. et al.. 1,4-Dioxane: Emerging technologies for an emerging contaminant. Remediation Journal 29, 49-63 (2019).

8.Patton, S. et al.. Impact of the Ultraviolet Photolysis of Monochloramine on 1,4-Dioxane Removal: New Insights into Potable Water Reuse. Environmental Science \& Technology Letters 4, 26-30 (2016).

9.Chuang, Y., Chen, S., Chinn, C. J. \& Mitch, W. A. Comparing the UV/Monochloramine and UV/Free Chlorine Advanced Oxidation Processes (AOPs) to the UV/Hydrogen Peroxide AOP Under Scenarios Relevant to Potable Reuse. Environmental Science \& Technology 51, 13859-13868 (2017).

10.Li, W. et al.. UV Photolysis of Chloramine and Persulfate for 1,4Dioxane Removal in Reverse-Osmosis Permeate for Potable Water Reuse. Environmental Science \& Technology 52, 6417-6425 (2018). 
11.Aitchison, E. W., Kelley, S. L., Alvarez, P. J. J. \& Schnoor, J. L. Phytoremediation of 1,4-Dioxane by Hybrid Poplar Trees. Water Environment Research 72, 313-321 (2000).

12.Oberle, D., Crownover, E. \& Kluger, M. In SituRemediation of 1,4Dioxane Using Electrical Resistance Heating. Remediation Journal 25, 35-42 (2015).

13.Patton, S., Romano, M., Naddeo, V., Ishida, K. P. \& Liu, H. Photolysis of Mono- and Dichloramines in UV/Hydrogen Peroxide: Effects on 1,4Dioxane Removal and Relevance in Water Reuse. Environmental Science \& Technology (2018) doi:10.1021/acs.est.8b01023.

14. Tabaï, A., Bechiri, O. \& Abbessi, M. Study of the degradation of a toxic dye by the catalytic system (H1.5Fe1.5P2W12Mo6061 22H2O)/H2O2. Euro-Mediterranean Journal for Environmental Integration 2, (2017). 